\title{
Reabilitação da memória em idosos com queixas mnemônicas e sintomas depressivos: estudo piloto não controlado
}

\author{
Tania Maria Netto \\ Universidade Federal do Rio de Janeiro \\ Rochele Paz Fonseca \\ Pontificia Universidade Católica do Rio Grande do Sul \\ Jesus Landeira-Fernandez \\ Universidade Estácio de Sá e Pontificia Católica do Rio de Janeiro
}

\begin{abstract}
Resumo
O presente estudo piloto investigou o efeito terapêutico de um programa de reabilitação da memória (RM), com avaliações pré e pós-intervenção, em um grupo de idosos com queixas mnemônicas e sintomas sugestivos de depressão. A amostra foi composta por sete idosos entre 65 a 80 anos de idade, acima de sete anos de estudo formal. A RM consistiu de 24 sessões, realizadas duas vezes por semana, cada com duração de 90 minutos. Esta intervenção incluiu técnicas de aprendizagem explícita e implícita, com estratégias mnemônicas internas e externas. Comparou-se o desempenho cognitivo pré e pós-intervenção pelo Teste Wilcoxon. Houve redução nas queixas de memória e nos sintomas sugestivos de depressão, aumento da velocidade de processamento atencional e aprimoramento da memória de trabalho. Sugere-se que este estudo seja replicado em amostras maiores, em grupos com déficits objetivos de memória e depressão clinicamente diagnosticada, comparados a grupos controles.
\end{abstract}

Palavras-chave: reabilitação; memória; depressão; idoso.

\begin{abstract}
Memory rehabilitation of elderly adults with mnemonic complaints and depressive symptoms: a pilot study. The present pilot study investigated the therapeutic effect of a memory rehabilitation program (MR), with pre and post-intervention assessment, in a group of elderly patients with mnemonic complaints and suggestive symptoms of depression. The sample was composed of seven older adults with ages between 65 to 80 years and formal education above seven years. The MR consisted of 24 sessions, twice weekly and each with 90 minutes duration. The intervention included implicit and explicit learning techniques, with internal and external mnemonic strategies. The cognitive performance, pre and post-interventions, were compared by the Wilcoxon test. There were reductions of suggestive symptoms of depression and of memory complaints, increase of attentional processing speed and improvement of working memory. This study should be replicated in larger samples, as well as, in groups with objective memory deficits and clinically diagnosed depression, compared to control groups.
\end{abstract}

Keywords: rehabilitation; memory; depression; elderly.

$\mathrm{O}$ presente estudo piloto aborda a temática reabilitação neuropsicológica da memória, em uma amostra de idosos com queixas de esquecimento e de sintomas sugestivos de depressão. A importância desta temática está relacionada ao recente crescimento da população idosa e ao aumento da longevidade, que pode resultar em aumento da prevalência de neuropatologias com possível comprometimento da memória, e/ou, até mesmo, de transtornos psiquiátricos, como a depressão. Este panorama apresenta a necessidade de intervenções neuropsicológicas que possam auxiliar a tratar, reabilitar, estabilizar ou até mesmo retardar a evolução dessas patologias para quadros mais graves em idosos.
Nas últimas décadas, o envelhecimento da população vem crescendo de forma acelerada, não só no Brasil, mas também no mundo. Em 2007, havia 650 milhões de pessoas acima de 60 anos no mundo; atualmente, há uma previsão de que em 2050 esta população chegará a dois bilhões (WHO, 2007). No Brasil, em 2010, a população acima de 65 anos foi registrada em 14 milhões (IBGE, 2010). No ano de 2008, existiam 24,7 idosos para 100 crianças recém-nascidas a 14 anos de idade. Em 2050 é previsto que haja 172,7 idosos para cada criança nessa mesma faixa etária (IBGE, 2008). Esse aumento da população idosa geralmente traz implicações para diversas áreas, especialmente na da saúde mental, tais como alterações cognitivas coexistentes 
ou associadas aos sintomas ou transtornos depressivos. Tais quadros podem interferir na realização das atividades diárias do idoso e, consequentemente, prejudicar sua qualidade de vida e de seus familiares.

Nesse contexto, vários estudos longitudinais e transversais têm evidenciado com frequência a coexistência de sintomas ou de transtornos depressivos e de queixas mnemônicas ou de desempenho rebaixado de idosos em avaliação neuropsicológica. Frente a estes achados, diferentes hipóteses explicativas têm sido sugeridas, tais como as ocorrências de queixas ou comprometimentos cognitivos: (1) podem ser decorrentes de sintomas ou transtornos depressivos (Dotson, Resnick, \& Zonderman, 2008; Minett, Silva, Ortiz, \& Bertolucci, 2008); (2) podem ter percursos e resultados heterogêneos, persistindo ou evoluindo para declínios mais acentuados, após o tratamento bem sucedido da depressão (Bhalla et al., 2006; Dierckx, Engelborghs, de Raedt, de Deyn, \& Ponjaert-Kristoffersen, 2007; Royall, Palmer, Chiodo, \& Polk, 2011); ou (3) podem estar relacionados ao início de doenças que causam demências, como doença cerebrovascular e doença de Alzheimer (Butters et al., 2008; Woolley, Khan, Murthy, Miller, \& Rankin, 2011). É possível que mais de uma dessas hipóteses expliquem a relação entre sintomas ou transtornos depressivos e queixas ou alterações mnemônicas, dificultando, muitas vezes, a acurácia diagnóstica, a intervenção terapêutica e o prognóstico desses quadros (Ávila \& Bottino, 2006). Dessa forma, queixas mnemônicas coexistentes ou associadas a sintomas sugestivos de depressão podem ser os primeiros indícios de um quadro mais grave, tais como declínio cognitivo leve e algum tipo de demência, principalmente a causada pela doença de Alzheimer (Dierckx et al., 2007; Royall et al., 2011; Woolley et al., 2011). Talvez estas queixas mnemônicas coexistentes ou associadas à depressão encontrem-se num estágio em que ainda possam ser revertidas ou estacionadas e não chegaram a um quadro complexo de comprometimentos mnemônicos.

A depressão no idoso, acompanhada de alterações cognitivas, quando tratada logo no início torna-se um quadro frequentemente reversível. No entanto, quando isso não acontece, pode resultar em comprometimentos cognitivos mais complexos, aumentar o risco de desenvolver outras doenças, além de contribuir para mudanças neurológicas na reorganização cerebral decorrente da neuroplasticidade negativa (Alexopoulos et al., 2002).

Dentre as alterações cognitivas mais prevalentes em quadros depressivos no idoso, destacam-se as mnemônicas (Crocco, Castro, \& Loewenstein, 2010; Köhler, Thomas, Barnett, \& O’Brien, 2010), atencionais (Ávila \& Bottino, 2006; Dotson et al., 2008; Rapp, Dahlman, Grossman, Haroutunian, \& Gorman, 2005), e/ou executivas, tais como déficits de velocidade de processamento e flexibilidade cognitiva (Ávila \& Bottino, 2006; Dotson et al., 2008; Laks \& Engelhardt, 2010). Em relação aos comprometimentos mnemônicos, a literatura tem mostrado com maior frequência déficits na memória de trabalho (MT) (Delaloye et al., 2008; Laks, \& Engelhardt, 2010) e/ou na memória episódica (Delaloye et al., 2008; Köhler et al., 2010).

De acordo com Wilson, Cockburn, Baddeley e Hiorns (1989) e Wilson e Evans (1996), o conceito de reabilitação cognitiva aplica-se a qualquer estratégia ou técnica de intervenção, que visa a habilitar um indivíduo que sofreu lesão cerebral e auxiliar seus familiares a lidar com comprometimentos cognitivos decorrentes da lesão. Seu objetivo mais importante é promover o máximo possível de funcionalidade do paciente, reduzindo suas incapacidades e reintegrando-o socialmente, podendo, assim, melhorar sua qualidade de vida. A reabilitação neuropsicológica, mais especificamente a mnemônica, tem sido cada vez mais valorizada na literatura especializada (Novoa, Juárez, \& Nebot, 2008; Willis et al., 2006). Junto a esta reabilitação, o modelo mais empregado tem sido o de processamento mnemônico entre sistemas múltiplos de memória (para uma revisão, Baddeley, 2009; Tulving, 1972). Programas baseados neste modelo buscam oferecer uma intervenção conduzida a partir da identificação de sistemas alterados que podem ser compensados pela estimulação dos sistemas preservados (Wilson \& Gracey, 2009). Nesse âmbito, a indicação de reabilitação neuropsicológica mnemônica pode ser uma solução promissora e de ordem preventiva.

Estudos internacionais têm investigado o efeito de programas de intervenções mnemônicas na melhoria de queixas de memória (Craik et al., 2007; Tsai, Yang, Lan, \& Chen, 2008), e/ou de depressão (Wolinsky et al., 2009a; Wolinsky et al., 2009b), em idosos independentes e sem demências. Dentre estes estudos, os que verificaram efeito terapêutico desses programas sob queixas de memória apresentaram resultados promissores. No entanto, aqueles que investigaram o efeito de reabilitação mnemônica sob suspeita de depressão clínica não apresentaram diferenças significativas na recuperação dos sintomas depressivos.

Em relação aos estudos nacionais, a reabilitação neuropsicológica tem sido uma temática ainda relativamente pouco explorada. No entanto, foram encontrados estudos que investigaram o efeito da reabilitação mnemônica sob déficits na memória e sintomas depressivos tanto em amostras de idosos com doença de Alzheimer (Ávila et al., 2004; Da-Silva et al., 2011) quanto com declínio cognitivo leve (Brum, Forlenza, \& Yassuda 2009). Achados dos estudos de Brum et al. (2009) e Da-Silva et al. (2011) apresentaram reduções significativas nos sintomas depressivos. Em contrapartida, os resultados do estudo de Ávila et al. (2004) mostraram apenas pequena melhora nos sintomas de depressão. Além desses estudos, outros foram realizados com amostras de idosos saudáveis. Esses estudos investigaram os efeitos de treinamentos referentes à memória episódica sob o uso de uma única estratégia ensinada e da velocidade de processamento (Carvalho, Neri, \& Yassuda, 2010); sob queixas de memória, depressão e outras variáveis (Yassuda, Batistoni, Fortes \& Neri, 2006); e o efeito de um treinamento referente à atenção, memória e funções executivas, sob a qualidade de vida e o bem-estar dos idosos (Irigaray, Schneider, \& Gomes, 2011). Estes estudos apresentaram resultados importantes em relação ao aprimoramento mnemônico, qualidade de vida, melhora da depressão e redução de queixas mnemônicas.

Pouco se sabe sobre os efeitos terapêuticos de reabilitação mnemônica em idosos brasileiros, com queixas de memória e sintomas sugestivos de depressão. Estudos nessa área, além de possuírem aplicabilidade clínica, podem também auxiliar na promoção de aumento da funcionalidade do idoso, melhorando sua qualidade de vida e de seus familiares. Nesse contexto, o presente estudo piloto teve como objetivo verificar o efeito 
terapêutico de um programa de Reabilitação da Memória (RM), em um grupo de idosos com queixas mnemônicas e sintomas sugestivos de depressão. A partir desse pressuposto as seguintes hipóteses foram formuladas: 1) O RM propiciará mudanças no processamento cognitivo das funções mnemônicas (MT e episódica) e em algumas habilidades a elas relacionadas (atenção concentrada e velocidade de processamento); além disso, 2) minimizará queixas mnemônicas, assim como 3) reduzirá sintomas sugestivos de depressão.

\section{Método}

\section{Participantes}

A amostra inicial deste estudo piloto consistiu em 14 idosos, quatro foram excluídos por não terem concluído a avaliação pré-intervenção (linha de base). Após o início da reabilitação, mais três participantes foram excluídos por não terem concluído as sessões de intervenção (um por desistência e dois por complicações de saúde). Todos eram destros, falantes nativos do Português Brasileiro e procedentes do Rio de Janeiro.

De acordo com os dados sociodemográficos e de saúde, as idades (I), anos de estudo formal (EF) e medicamentos (M) dos participantes $(\mathrm{P})$ foram os seguintes: $\mathrm{P} 1, \mathrm{I}=69$ anos, $\mathrm{EF}$ $=7$ anos e $\mathrm{M}=$ Não tomava nenhum remédio; $\mathrm{P} 2, \mathrm{I}=68$ anos, $\mathrm{EF}=12$ anos e $\mathrm{M}=$ Nenhum; $\mathrm{P} 3, \mathrm{I}=70$ anos, $\mathrm{EF}=18$ anos e $\mathrm{M}=$ Nenhum; $\mathrm{P} 4, \mathrm{I}=77$ anos, $\mathrm{EF}=15$ anos e $\mathrm{M}=$ Citalopram (Antidepressivo); $\mathrm{P} 5, \mathrm{I}=78$ anos, $\mathrm{EF}=13$ anos e $\mathrm{M}=$ Nenhum; $\mathrm{P} 6, \mathrm{I}=78$ anos, $\mathrm{EF}=18$ anos e $\mathrm{M}=$ Nenhum; $\mathrm{P} 7, \mathrm{I}=81$ anos, $\mathrm{EF}=9$ anos e $\mathrm{M}=$ Nenhum Dentre estes sete idosos, apenas um, do sexo feminino, estava fazendo uso de antidepressivo e mesmo assim apresentou queixas de memória e sintomas sugestivos de depressão moderada conforme a Escala de Depressão Geriátrica (GDS-15) (Yesavage et al., 1983, versão adaptada por Almeida, \& Almeida, 1999). Quanto ao sexo, esta amostra constituiu-se de seis mulheres e um homem. Todos eles eram aposentados e apresentaram queixas de memória e sintomas sugestivos de depressão. Nenhum desses participantes apresentou pontuações no Mini Exame do Estado Mental (MEEM) (Folstein, Folstein, \& McHugh, 1975, versão adaptada por Almeida, 1998) igual ou abaixo do ponto de corte (24), sugerindo ausência de possíveis déficits cognitivos indicativos de quadro demencial. Em relação à GDS-15, na pré-avaliação o escore mínimo foi 6 e o máximo 14 , indicando nível leve moderado e grave $(\mathrm{P} 1=8, \mathrm{P} 2=10, \mathrm{P} 3$ $=13, \mathrm{P} 4=6, \mathrm{P} 5=9, \mathrm{P} 6=6, \mathrm{P} 7=14$ ) de sintomas sugestivos de depressão.

O recrutamento dessa amostra de conveniência foi realizado por anúncios em jornais de bairros da zona sul do Rio de Janeiro. Para serem incluídos na amostra, os idosos deveriam apresentar na avaliação pré-intervenção: 1) queixas de memória examinadas pelo Questionário de Auto-relato de Queixas de Memória, formado por 13 perguntas com respostas fechadas - sim ou não (adaptado de Strauss, Sherman, \& Spreen, 2006); 2) presença de sintomas sugestivos de depressão no idoso (pontuação $\geq 5$ na GDS-15) (Castelo et al., 2010); 3) e ausência de possíveis déficits cognitivos (pontuação $\geq 24$ pontos no MEEM). Os critérios de exclusão consistiram na ausência de auto-relato de possíveis transtornos psiquiátricos, diagnóstico prévio de depressão maior e histórico, prévio ou atual, de alcoolismo ou uso de drogas ilícitas e de benzodiazepínicos, bem como comprometimentos neurológicos e déficits auditivos e visuais não corrigidos.

\section{Procedimentos e instrumentos}

Após o Termo de Consentimento Livre e Esclarecido ter sido assinado por todos os participantes, mediante aprovação do Comitê de Ética em Pesquisa da Instituição (número 02 / 2008), conduziram-se procedimentos em três etapas: (1) avaliação pré-intervenção (linha de base ou pré-teste); 2) programa de TM; e 3) avaliação pós-intervenção. As avaliações pré e pósintervenção foram administradas em três sessões, com duração de uma hora cada e com intervalo máximo de três semanas. No pré-teste, os participantes passaram inicialmente por uma préseleção, seguidos de três sessões de avaliações psicológicas e neuropsicológicas. Na fase de pré-seleção, uma breve entrevista foi realizada com cada idoso por meio de contato telefônico, para verificar idade, escolaridade, e alguns critérios de inclusão, tais como queixas de memória e sintomas depressivos. Na primeira sessão de avaliação, administraram-se o MEEM, teste de rastreamento de possíveis déficits cognitivos (Almeida, 1998), entrevista semi-estruturada e GDS-15 (Almeida \& Almeida, 1999). Aqueles que cumpriram os critérios de inclusão foram avaliados em uma segunda sessão com subtestes da Escala de Inteligência Wechsler para Adultos (WAIS-III): 1) Dígitos de Ordem Direta (avaliou atenção e memória de curto-prazo), 2) Dígitos de Ordem Indireta (memória de trabalho), 3) Informação (memória semântica e habilidades de linguagem), e 4) Sequência de Números e Letras (memória de trabalho) (Nascimento, 2004), e por fim o Teste de Aprendizagem Auditivo-Verbal de Rey que mensurou aprendizagem, memória de curto-prazo e de longo prazo (RAVLT, Malloy-Diniz, Cruz, Torres, \& Cosenza, 2000; Malloy-Diniz, Lasmar, Gazinelli, Fuentes, \& Salgado, 2007). $\mathrm{Na}$ terceira sessão, os participantes continuaram a ser avaliados pelos subtestes de atenção, memória e funções executivas do Instrumento de Avaliação Neuropsicológica Breve NEUPSILIN (Fonseca, Salles, \& Parente, 2008c, 2009) e pelos subtestes de Discurso Narrativo da Bateria Montreal de Avaliação da Comunicação - Bateria MAC, (Fonseca, Parente, Côté, Ska, \& Joanette, 2008a, 2008b). Ressalta-se que a bateria de testes neuropsicológicos foi administrada e pontuada por um psicólogo treinado diferente daquele que conduziu a reabilitação.

\section{Programa de treinamento da memória - TM}

O TM consistiu em 24 sessões, que foram realizadas duas vezes por semana, cada uma com duração de 90 minutos. $\mathrm{O}$ programa ocorreu de forma grupal e foi embasado em procedimentos explícitos e implícitos. Na reabilitação dos componentes específicos da memória foram incluídas as seguintes técnicas mnemônicas explícitas: 1) Facilitação da memória explícita residual com suporte cognitivo - embasada na codificação e na recuperação de informações, facilitando o aumento da capacidade de aprendizagem e da manipulação mental de informações adquiridas (Holderbaum, Rinaldi, Brandão, \& Parente, 2006); 2) Categorização - colocar estímulos em suas respectivas categorias (por exemplo - filme 
de ficção científica ou romântico) (Ávila et al., 2004; Craik et al., 2007); 3) Associação - associar estímulo a algo relevante (Ávila et al., 2004; Craik et al., 2007); 4) Criação de história - criar histórias breves relacionada aos estímulos (Craik et al., 2007); 5) Leitura de artigos (Craik et al., 2007); 6) Estratégias compensatórias com o auxílio de suporte externo - utilização de objetos (agendas, diários, despertadores, pagers, calendários dentre outros) como auxiliares da memória (Kapur, 2009). Além dessas técnicas, também foram empregadas outras para estimular a memória implícita, tais como, 7) Método de recuperação espaçada - evocação de determinada informação memorizada durante pequenos intervalos de tempo e de acordo com o progresso do indivíduo, o espaçamento dessa informação aumenta gradativamente (Glisky \& Glisky, 2008; Wilson, 2009); 8) Método de apagamento de pistas - pistas são fornecidas para facilitar a evocação de determinadas tarefas ou informações. $\mathrm{O}$ número de pistas é reduzido gradativamente até serem eliminadas (Wilson, 2009); 9) Aprendizagem sem erro - durante o processo de aprendizagem de novas informações, erros são evitados ou reduzidos de forma significativa (Baddeley \& Wilson, 1994; Wilson, 2009).

Nas primeiras sessões houve introdução entre os membros do grupo e palestras educativas sobre a memória no envelhecimento e sobre o programa de reabilitação. A partir da quarta à vigésima segunda sessão, exceto as duas últimas, que foram de fechamentos, houve uma rotina em relação ao emprego de estratégias e técnicas mnemônicas, variando apenas os estímulos a serem processados em cada sessão. Ressalta-se que a técnica de Aprendizagem sem Erro foi empregada durante todas as etapas das sessões. Os participantes foram orientados pelo neuropsicólogo para não adivinharem respostas das tarefas e quando não as soubessem deveriam pedir pistas evitando, assim, consolidar respostas erradas e também se sentirem constrangidos perante os outros membros do grupo, quando errassem.

\section{Análise de dados}

O resultado do TM foi avaliado por meio da comparação entre o desempenho neuropsicológico pré- e pós-intervenção e os escores médios obtidos pelos instrumentos neuropsicológicos foram analisados pelo teste estatístico, não-paramétrico, Wilcoxon. Estes dados foram analisados pelo software SPSS $12.0, p \leq 0,05$. Os escores $Z$ foram calculados para verificar possíveis déficits cognitivos (ponto de corte sugerido por Schoenberg et al., 2006, $Z \leq-1,50$ ) dos participantes na linha de base e assim identificar efeito de teto. Para complementar a estatística inferencial, calculou-se o Tamanho do Efeito (TE) terapêutico dos parâmetros que apresentaram uma significância estatística no Wilcoxon, pelo teste $d$ (Cohen, 1988). Os valores do TE foram interpretados de acordo com a recomendação do critério de Cohen (1988), do qual 0,2 é indicativo de um valor do TE pequeno; 0,5 do TE médio e 0,8 do TE grande (Dancey \& Reidy, 2006).

\section{Resultados}

Na Tabela 1, são apresentados os resultados das escalas, questionários e testes neuropsicológicos com suas respectivas médias, desvios-padrão, valores máximos e mínimos dos escores obtidos nas avaliações neuropsicológicas, pré e pós-intervenção. Nesta Tabela 1, encontram-se também os respectivos escores $Z$ e os níveis de significância do teste Wilcoxon.

Conforme os resultados presentes na Tabela 1, pode-se observar uma diferença significativa na redução dos sintomas sugestivos de depressão pós-intervenção e das queixas de memória. Além dessas variáveis, pode-se também observar uma diferença significativa na diminuição do tempo de processamento atencional e um aumento no número de acertos em tarefa de exame predominante do componente executivo central da MT. Nos demais testes diferenças significativas não foram encontradas.

$\mathrm{O}$ escore $Z$ médio foi calculado para cada variável e apenas uma delas apresentou déficit na linha de base, no Teste de Aprendizagem Auditivo-verbal de Rey (RAVLT), escore A6 - número de palavras evocadas da lista A após interferência da lista $\mathrm{B}$ (escore $Z=-2,04$ ). Isto sugere que, em geral, nenhum participante apresentava déficits objetivos de memória na linha de base, mas sim queixas subjetivas.

A Tabela 2 apresenta os valores do TE terapêutico das variáveis que demonstraram diferenças significativas após o TM. Conforme se pode observar na Tabela 2, o $d$ de Cohen revelou que o impacto do TM foi maior nas variáveis GDS-15 e no Questionário de Auto-Relato de Queixas de Memória em comparação às variáveis relacionadas à MT e à velocidade de processamento de informação.

\section{Discussão}

Queixas de memória e sintomas sugestivos de depressão são geralmente frequentes em adultos idosos podendo evoluir para quadros mais graves. Muitas vezes este quadro pode ser revertido, após reabilitação mnemônica (Brum et al. 2009; Craik et al., 2007; Da-Silva et al., 2011; Tsai, Yang, Lan, \& Chen, 2008). Os resultados do presente estudo piloto apresentaram efeitos terapêuticos nas variáveis queixas de memória, sintomas sugestivos de depressão, executivo central, componente da MT, e velocidade de processamento em tarefa atencional. $\mathrm{O}$ efeito terapêutico (TE) foi maior nas medidas de sintomas sugestivos de depressão e queixas de memória. Deste modo, as hipóteses iniciais foram confirmadas, exceto em relação à melhora no desempenho dos idosos em tarefas da memória episódica.

No que se refere à primeira hipótese formulada "O TM propiciará mudanças no processamento cognitivo das funções mnemônicas (MT e episódica) e em algumas habilidades a elas relacionadas (atenção concentrada e velocidade de processamento)", melhoras significativas foram apresentadas no desempenho das tarefas que avaliaram o executivo central e a velocidade de processamento da atenção concentrada, exceto aquelas que avaliaram a memória episódica. O TM foi estruturado de forma que as mesmas técnicas mnemônicas, citadas no método, fossem usadas e integradas aos seus devidos processamentos verbais em todas as sessões do TM. Vale ressaltar que todas as sessões foram realizadas com diferentes estímulos apresentados em forma de imagens e processados verbalmente. Durante as sessões, participantes precisavam 
Tabela 1

Resultado da análise comparativa intragrupos quanto aos sintomas sugestivos de depressão, queixas de memória e desempenho neuropsicológico nos periodos pré e pós-intervenção

\begin{tabular}{|c|c|c|c|c|c|}
\hline \multirow{3}{*}{\multicolumn{2}{|c|}{ Variáveis }} & \multicolumn{4}{|c|}{ Grupo experimental } \\
\hline & & Pré-intervenção & Pós-intervenção & & \\
\hline & & $\begin{array}{l}\text { Média } \pm d p \\
(\text { Min-Max) }\end{array}$ & $\begin{array}{l}\text { Média } \pm d p \\
(\text { Min-Max) }\end{array}$ & $Z$ & $p$ \\
\hline \multirow{8}{*}{ 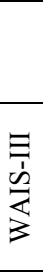 } & MEEM & $27,29 \pm 1,49(25-29)$ & $28,57 \pm 1,61(26-30)$ & $-1,279$ & 0,201 \\
\hline & GDS-15 & $9,43 \pm 3,15(6-14)$ & $3,29 \pm 2,69(0-8)$ & $-2,366$ & 0,018 \\
\hline & Auto-relato de queixas de memória & $6,20 \pm 1,48(4-8)$ & $3,40 \pm 1,14(2-5)$ & $-2,041$ & 0,041 \\
\hline & SNL & $7,71 \pm 2,05(5-11)$ & $6,00 \pm 2,23(4-9)$ & $-1,80$ & 0,071 \\
\hline & Dígitos OD & $8,43 \pm 2,14(5-12)$ & $7,57 \pm 1,39(5-9)$ & $-1,89$ & 0,059 \\
\hline & Dígitos OI & $5,00 \pm 1,73(3-8)$ & $4,86 \pm 1,95(2-7)$ & $-0,37$ & 0,705 \\
\hline & Dígitos OD/OI & $13,43 \pm 3,20(8-17)$ & $12,43 \pm 3,10(7-16)$ & $-1,72$ & 0,084 \\
\hline & Informação & $17,00 \pm 6,44(7-22)$ & $15,14 \pm 6,66(3-22)$ & $-0,73$ & 0,461 \\
\hline \multirow{8}{*}{ 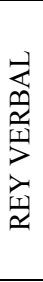 } & Palavras em A1 & $5,43 \pm 1,81(4-9)$ & $6,86 \pm 2,41(3-10)$ & $-1,19$ & 0,232 \\
\hline & Palavras em A2 & $7,14 \pm 2,11(5-10)$ & $8,00 \pm 2,7(4-11)$ & $-0,85$ & 0,394 \\
\hline & Palavras em A3 & $7,71 \pm 2,56(4-11)$ & $8,86 \pm 2,47(5-11)$ & $-1,28$ & 0,190 \\
\hline & Palavras em A4 & $9,43 \pm 1,71(7-12)$ & $9,57 \pm 2,69(5-12)$ & $-0,17$ & 0,864 \\
\hline & Palavras em A5 & $9,43 \pm 2,14(6-12)$ & $9,57 \pm 2,50(5-12)$ & $-0,18$ & 0,854 \\
\hline & Palavras em A6 & $7,43 \pm 2,22(5-11)$ & $8,43 \pm 3,15(5-13)$ & $-1,12$ & 0,262 \\
\hline & Palavras na $\sum \mathrm{A} 1-\mathrm{A} 5$ & $39,29 \pm 8,99(26-52)$ & $42,86 \pm 11,37(23-55)$ & $-0,94$ & 0,345 \\
\hline & Reconhecimento & $13,71 \pm 1,7(11-15)$ & $12,57 \pm 3,50(5-15)$ & $-1,09$ & 0,273 \\
\hline \multirow{11}{*}{$\begin{array}{l}\text { Z } \\
\text { 品 } \\
\text { 号 } \\
\text { 夏 }\end{array}$} & AC - Cont. Inversa & $19,43 \pm 1,13(17-20)$ & $18,43 \pm 3,3(11-20)$ & $-1,34$ & 0,180 \\
\hline & AC - Cont. inversa (tempo) & $\begin{array}{l}28,71 \pm 13,94 \\
(19,53-59,17)\end{array}$ & $\begin{array}{c}20,42 \pm 4,75 \\
(13,12-29,19)\end{array}$ & $-2,19$ & $\mathbf{0 , 0 2 8}$ \\
\hline & Repetição de dígitos (omissões) & $1,71 \pm 1,38(0-3)$ & $0,71 \pm 0,75(0-2)$ & $-0,25$ & 0,102 \\
\hline & MT - Ordenamento de Dígitos & $6,57 \pm 1,81(5-9)$ & $8,14 \pm 1,67(6-10)$ & $-2,26$ & 0,024 \\
\hline & MT - SAPS & $12,71 \pm 1,49(10-14)$ & $14,14 \pm 5,11(7-19)$ & $-0,95$ & 0,339 \\
\hline & MV - Evocação imediata & $4,29 \pm 1,97(1-7)$ & $4,57 \pm 1,13(4-7)$ & $-0,53$ & 0,593 \\
\hline & MV- Evocação tardia & $1,57 \pm 1,9(0-5)$ & $0,71 \pm 1,89(0-5)$ & $-1,60$ & 0,109 \\
\hline & MSLP & $4,86 \pm 0,37(4-5)$ & $4,71 \pm 0,48(4-5)$ & $-0,57$ & 0,564 \\
\hline & MVCP & $2,86 \pm 0,37(2-3)$ & $2,86 \pm 0,37(2-3)$ & $-0,00$ & 1,000 \\
\hline & Memória Prospectiva & $0,86 \pm 0,69(0-2)$ & $1.00 \pm 0,81(0-2)$ & $-1,00$ & 0,317 \\
\hline & Habilidades Aritméticas & $8,00 \pm 0,00(8-8)$ & $7,71 \pm 0,75(6-8)$ & $-1,00$ & 0,317 \\
\hline \multirow{4}{*}{$\sum_{\Sigma}^{U}$} & DN (parcial) - Inf. essenciais & $12,86 \pm 4,59(5-17)$ & $13,43 \pm 4,03(5-18)$ & $-0,08$ & 0,932 \\
\hline & DN (parcial) - Inf. presentes & $17,57 \pm 6,10(7-23)$ & $17,86 \pm 5,21(7-22)$ & 0,00 & 1,000 \\
\hline & DN (integral) & $7,43 \pm 3,30(3-11)$ & $8,29 \pm 2,69(3-11)$ & $-0,60$ & 0,546 \\
\hline & DN (questões) & $10,14 \pm 2,03(6-12)$ & $9,71 \pm 2,05(7-12)$ & $-0,74$ & 0,457 \\
\hline
\end{tabular}

Nota. A comparação intragrupo pré e pós-intervenção com o Teste Wilcoxon foi efetuada com base nos escores brutos por estes apresentarem maior variabilidade do que os escores $Z$; GDS-15 = Escala de Depressão Geriátrica; $\mathrm{SNL}=$ Sequência de Números e Letras; OD = Ordem direta; OI = Ordem indireta; $\mathrm{AC}=$ Atenção concentrada; $\mathrm{MT}=$ Memória de trabalho; SAPS $=$ Span auditivo de palavras em sentenças; $\mathrm{MV}=$ memória verbal; MSLP = Memória semântica de longo prazo; $\mathrm{MVCP}=$ Memória visual de curto prazo; $\mathrm{MAC}=$ Bateria Montreal de Avaliação da Comunicação; DN = Discurso narrativo. O nível de significância foi de $p \leq 0,05 . Z$ : Wilcoxon test.

Tabela 2

Tamanho do efeito do PRM de idosos com queixas de memória e sintomas sugestivos de depressão apresentada por instrumento

\begin{tabular}{lc}
\hline & Tamanho do Efeito \\
\hline GDS-15 & 1,44 \\
Auto-relato de Queixas de Memória-Total & 1,43 \\
Memória de Trabalho - Ordenamento ascendente de dígitos & 0,84 \\
Atenção - Tempo total - Contagem Inversa & 0,76 \\
\hline
\end{tabular}

Nota: GDS-15 = Escala de Depressão Geriátrica com 15 itens

manter as informações aprendidas online, pois eram usadas com frequência. Esses procedimentos podem ter contribuído na melhora do desempenho dos idosos na tarefa ordenamento de dígitos (NEUPSILIN), pós-intervenção, na qual o executivo central foi avaliado.

Uma possível explicação para apenas o componente executivo central da MT ter sido aprimorado, pode ser a obtenção de pouco treinamento cognitivo dos outros componentes da MT, em virtude do programa não ter sido desenvolvido especificamente para este tipo de memória. Em concordância com essa explicação, um estudo de idosos octogenários com a mesma duração do presente estudo, três meses, também apresentou melhora no desempenho de apenas uma tarefa da MT, o componente esboço visuoespacial (Buschkuehl et al., 
2008). Em contrapartida, o estudo de Craik et al. (2007), que avaliou os efeitos de três programas (Mnemônico, Psicossocial e Manejo de Metas) no desempenho da memória, não encontrou diferenças significativas em tarefas referentes à MT em nenhum desses treinamentos, até mesmo no mnemônico que usou técnicas similares ao presente estudo. Tal achado pode ter ocorrido devido ao fato do treinamento mnemônico ter durado somente quatro semanas e não como no presente estudo.

Em relação à memória episódica, diferenças significativas não foram encontradas pré e pós-intervenção, provavelmente pelo fato dessa função não ter sido trabalhada diretamente. Além disso, o tamanho reduzido da amostra final, típico de estudos piloto de reabilitação neuropsicológica (Ávila et al., 2004), pode ter contribuído para este achado. Ao contrário desses resultados, dois estudos que instruíram seus participantes com técnicas explícitas específicas para evocar listas de palavras (Belleville et al., 2006) e pequenas narrativas (Unverzagt et al., 2007) relataram diferenças significativas no desempenho dos idosos em tarefas de memória episódica quando comparados com controles.

Dentre as alterações cognitivas que ocorrem durante o envelhecimento, a velocidade de processamento tem sido uma das que tem recebido mais atenção recentemente. Esta função interfere no funcionamento eficiente de operações cognitivas complexas, tais como MT e memória episódica (Acevedo \& Loewenstein, 2007). No presente estudo, o único componente das funções executivas que apresentou efeito de transferência foi a velocidade de processamento atencional. Isto pode ter ocorrido devido à redução dos sintomas sugestivos de depressão, pois esta função é uma das mais afetadas em quadros depressivos (Ávila \& Botino, 2006; Laks \& Engelhardt, 2010). Adicionalmente, talvez as tarefas de avaliação utilizadas neste estudo não tenham sido sensíveis suficientes para avaliar o processo de transferência para outros componentes das funções executivas. A inclusão de tarefas ecológicas, assim como de medidas de escalas funcionais, na avaliação pré e pós-intervenção poderiam ter revelado resultados mais favoráveis. Ressalta-se que esses achados referentes às alterações cognitivas devem ser considerados com muita cautela, visto que podem representar um efeito de retestagem. Além disso, as múltiplas comparações efetuadas devem ser replicadas em próximos estudos com grupo controle para reduzir a possibilidade de achados ao acaso.

A segunda hipótese "O TM minimizaria queixas mnemônicas" foi confirmada. Tal resultado pode estar associado à redução dos sintomas sugestivos de depressão, concordando, assim, com outros estudos transversais realizados em amostras de idosos, que sugeriram associação entre queixas subjetivas de memória e sintomas depressivos (Bolla, Lindgren, Bonaccorsy, \& Bleecker, 1991; Minett et al., 2008). Além disso, estimulações de diferentes sistemas da memória e a convivência social em grupo durante o TM podem também ter contribuído na melhora das queixas mnemônicas. Em concordância com esses dados, estudos que investigaram o efeito de treinamentos mnemônicos realizados de forma individual e grupal observaram que treinamentos realizados em formas grupais promoveram mais efeitos positivos nos desempenhos em tarefas mnemônicas do que os individuais (Hasting \& West 2009).

Por fim, a terceira hipótese "O TM reduzirá sintomas sugestivos de depressão" também foi confirmada. Esta redução de sintomas sugestivos de depressão pode ter ocorrido devido à interação entre os membros da amostra do presente estudo, proporcionando a eles aumento na convivência social e senso de universalidade (conscientização do idoso de que ele não é o único com queixas mnemônicas e sintomas sugestivos de depressão) no grupo (Yalom \& Leszcz, 2005). Embora alguns estudos tenham obtido resultados similares (Brum et al, 2009; Da-Silva et al., 2011; Winocur et al., 2007), outros não relataram melhoras significativas em sintomas depressivos após treinamentos mnemônicos (Ávila et al., 2004; Wolinsky et al. 2009a; Wolinsky et al., 2009b). O uso de imagens de estímulos prazerosos decorrentes da lista de atividades em todas as sessões pode ter contribuído também na melhora dos sintomas sugestivos de depressão.

Foram encontrados três estudos brasileiros que verificaram efeitos de treinamentos mnemônicos sobre déficits de memória e sintomas depressivos. Suas amostras eram compostas de pacientes com demência do tipo Alzheimer (Ávila et al., 2004; Da-Silva et al., 2011) e declínio cognitivo leve (Brum et al., 2009). Dois destes estudos (Brum et al., 2009; Da-Silva et al., 2011) apresentaram redução significativa nos sintomas de depressão, quando avaliados pela Escala de Depressão Geriátrica (Yesavage et al., 1983), mesma escala usada neste presente estudo. Em contrapartida, no estudo de Ávila et al. (2004) relatou-se pequena melhora nos sintomas de depressão, sendo que esses foram avaliados pela Escala de Depressão Montgomery-Alsberg (Montgomery \& Alsberg, 1979) . Esses dados sugerem que a Escala de Depressão Geriátrica pode ser mais sensível do que a de Montgomery-Alsberg na avaliação de sintomas depressivos em idosos.

Aspectos metodológicos, como tamanho da amostra e ausência de um grupo controle, foram algumas das limitações que podem ser justificadas pela natureza piloto do presente estudo. Além dessas, outras limitações importantes foram a longa duração da avaliação neuropsicológica, podendo ser reduzida em futuros estudos, assim como a inclusão de um participante fazendo uso de antidepressivo. Vale ressaltar que mesmo com esse medicamento o participante ainda apresentou queixas de memória e sintomas sugestivos de depressão moderada.

Conclui-se que, após o TM, o resultado de maior significância foi a alteração do estado do humor e, por segundo, o relato da redução das queixas de memória, sugerindo possível envolvimento de questões emocionais e de autoimagem desses idosos sem demências. Em relação às hipóteses explicativas apresentadas na introdução, a que mais se adequou aos resultados do presente estudo foi "queixas ou comprometimentos mnemônicos podem ser decorrentes de sintomas ou transtornos depressivos". No que se refere ao TM, este parece ser um método replicável, coletivo e de baixo custo que pode ser facilmente empregado na clínica e em diferentes amostras, visando a reduzir sintomas sugestivos de depressão, bem como reabilitar e monitorar alterações cognitivas, especialmente mnemônicas em idosos sem demência. Sugere-se que futuros estudos sejam realizados com amostras maiores, empregando métodos de ensaio clínico randomizado, investigando questões emocionais e de auto-imagem desses idosos, bem como adicionando técnicas 
textuais mais complexas direcionadas à memória episódica. Seria interessante, ainda, conduzir estudos desse TM com amostras clinicamente diagnosticadas com depressão maior, e sinais objetivos de déficit de memória. Mais investigações sobre efeitos terapêuticos de programas de treinamento cognitivo e de reabilitação neuropsicológica devem ser conduzidas no Brasil para que programas e técnicas sejam disponibilizados à comunidade científica e clínica, proporcionando benefícios diretos aos idosos com alterações cognitivas e sintomas depressivos, melhorando assim a qualidade de vida de todos envolvidos.

\section{Referências}

Acevedo, A., \& Loewenstein, D. A. (2007). Nonpharmacological cognitive interventions in aging and dementia. Journal of Geriatric Psychiatry and Neurology, 20(4), 239-249.

Alexopoulos, G. S., Buckwalter, K., Olin, J., Martinez, R., Wainscott, C., \& Krishnan, K. R. R. (2002). Comorbidity of late life depression: an opportunity for research on mechanisms and treatment. Biological Psychiatry, 52(6), 543-558.

Almeida, O. P. (1998). Mini exame do estado mental e o diagnóstico de demência no Brasil. Arquivos de NeuroPsiquiatria, 56(3B), 605-612.

Almeida, O. P., \& Almeida, S. A. (1999). Confiabilidade da versão brasileira da Escala de Depressão em Geriatria (GDS) versão reduzida. Arquivos de NeuroPsiquiatria, 57(2B), 421-426.

Ávila, R., \& Bottino, C. M. C. (2006). Atualização sobre alterações cognitivas em idosos com síndrome depressiva. Revista Brasileira de Psiquiatria, 28(4), 316-320.

Ávila, R., Bottino, C. M. C., Carvalho, I. A. M., Santos, C. B., Seral, C., \& Miotto, E. C. (2004). Neuropsychological rehabilitation of memory deficits and activities of daily living in patients with Alzheimer's disease: a pilot study. Brazilian Journal of Medical and Biological Research, 37, 1721-1729.

Baddeley A. (2009) Working memory. In A. D. Baddeley, M. W. Eysenck, \& M. C. Anderson (Orgs.), Memory (pp. 41-68). New York: Psychology Press.

Baddeley, A., \& Wilson, B. (1994). When implicit learning fails: amnésia and the problem of error elimination. Neuropsychologia, 32, 53-68.

Belleville, S., Brigitte, G., Fontaine, F., Gagnon, L., Ménard, E., \& Gauthier, S. (2006). Improvement of episodic memory in persons with mild cognitive impairment and healthy older adults: evidence from a cognitive intervention program. Dementia and Geriatric Cognitive Disorders, 22, 486-499.

Bhalla, R., Butters, M., Mulsant, B., Begley, A., Zmuda, M., Schoderbeck, B., ... $\&$ Becker, J. (2006). Persistence of neuropsychologic deficits in the remitted state of late-life depression. American Journal of Geriatric Psychiatry, 14(5), 419-427.

Bolla, K. I., Lindgren, K. N., Bonaccorsy, C., \& Bleecker, M. L. (1991). Memory complaints in older adults. Fact or fiction? Archives of Neurology, 48(1), 61-64.

Brum, P. S., Forlenza, O. V., \& Yassuda, M. S. (2009). Cognitive training in older adults with Mild Cognitive Impairment. Dementia \& Neuropsychologia, 3(2), 124-131.

Buschkuehl, M., Jaeggi, S. M., Hutchison, S., Perrig-Chiello, P., Däpp, C., Müller, M., ... Perrig, W. J. (2008). Impact of working memory training on memory performance in old-old adults. Psychology and Aging, 23(4), 743-753.

Butters, M. A., Jeffrey, B., Young, B. A., Lopez, O., Aizenstein, H. J., Mulsant, B. H., Reynolds III, ... \& Becker, J. T. (2008). Pathways linking late-life depression to persistent cognitive impairment and dementia. Dialogues in Clinical Neuroscience, 10(3), 345-357.

Carvalho. F., Neri, A., \& Yassuda, M. (2010). Treino de memória episódica com ênfase em categorização para idosos sem demência e depressão. Psicologia: Reflexão e Crítica, 23(2), 317-323.
Castelo, M. S., Coelho-Filho, J. M., Carvalho, A. F., Lima, J. W. O., Noleto, J. C. S., Ribeiro, K. G., \& Siqueira-Neto, J. I. (2010). Validity of the Brazilian version of the Geriatric Depression Scale (GDS) among primary care patients. International Psychogeriatrics, 22(1), 109-113.

Cohen, J. (1988). Statistical power analysis for the behavioral sciences. Hillsdale: Lawrence Erlbaum Associates.

Craik, F. I. M., Winocur, G., Palmer, H., Binns, M. A., Edwards, M., Bridges, K., ... Stuss, D. T. (2007). Cognitive rehabilitation in the elderly: effects on memory. Journal of the International Neuropsychological Society, 13, $132-142$

Crocco, E. A., Castro, K., \& Loewenstein, D. A. (2010). How late-life depression affects cognition: neural mechanisms. Current Psychiatry Report, 12, 34-38.

Dancey, C., P., \& Reidy, J. (2006). Estatística sem matemática para psicólogos: usando SPSS para Windows. São Paulo: Artmed.

Da-Silva, S. L., Pereira, D. A., Veloso, F., Satler, C. E., Arantes, A., Guimarães, R. M. (2011). Programa de reabilitação neuropsicológica da memória aplicada à demência: um estudo não controlado intrasujeitos. Estudos de Psicologia (Campinas), 28(2), 229-240.

Delaloye, C., Baudois, S., de Bilbao, F., Remund, C. D., Hofer, F., Lamon, M., ... \& Giannakopoulos, P. (2008). Cognitive impairment in late-onset depression limited to a decrement in information processing resources? European Neurology, 60, 149-154.

Dierckx, E., Engelborghs, S., de Raedt, R., de Deyn, P. P., \& PonjaertKristoffersen, I. (2007). Mild cognitive impairment: what's in a name? Gerontology, 53, 28-35.

Dotson, V., Resnick, S., \& Zonderman, A. (2008). Differential association of concurrent, baseline, and average depressive symptoms with cognitive decline in older adults. American Journal Geriatric Psychiatry, 16(4), 318-330.

Fonseca, R. P., Parente, M. A. M. P., Côté, H., Ska, B., \& Joanette, Y. (2008a). Bateria montreal de Avaliação da Comunicação-Bateria MAC. São Paulo, Pró-Fono.

Fonseca, R. P., Parente, M. A. M. P., Côté, H., Ska, B., \& Joanette, Y. (2008b). Introducing a communication assessment tool to Brazilian speech therapists: the MAC Battery. Pro Fono, 20(4), 285-291.

Fonseca, R. P., Salles, J. F., \& Parente, M. A. M. P. (2008c). Development and content validity of the Brazilian Brief Neuropsychological Assessment Battery NEUPSILIN. Psychology \& Neuroscience, 1(1), 55-62.

Fonseca, R. P., Salles, J. F., \& Parente, M. A. M. P. (2009). Instrumento de Avaliação Neuropsicológica Breve NEUPSILIN. Porto Alegre: Vetor.

Folstein, M., Folstein, S., \& McHugh, P. (1975). Mini-mental state: a practical method for grading the cognitive state of patients for the clinician. Journal of Psychiatric Research, 12, 189-198.

Glisky, E., \& Glisky, M. (2008). Memory rehabilitation in older adults. In D. Stuss, G. Winocur, \& I. Robertson (Orgs.), Cognitive neurorehabilitation (pp. 541-561). Nova Iorque: Cambridge University Press

Hasting, E. C., \& West, R. L. (2009). The relative success of a self-help and a group-base memory training program for older adults. Psychology and Aging, 24(3), 586-594.

Holderbaum, C. S., Rinaldi, J., Brandão, L., \& Parente, M. A. M. P. (2006) A intervenção cognitiva para pacientes portadores de demência do tipo Alzheimer. In M. A. M. P. Parente (Org.), Cognição e envelhecimento (pp. 259-273). Porto Alegre: Artmed.

IBGE (2008). O país caminha velozmente rumo a um perfil demográfico cada vez mais envelhecido. Recuperado de $<$ http://www.ibge.gov.br/home/ presidencia/noticias/noticia_impressao.php?id_noticia=1272>.

IBGE (2010). Sinopse do Censo demográfico. Recuperado de <http: //www. censo2010.ibge.gov.br/sinopse/index.php?dados $=12 \& u f=00 \#$ topo piramide>.

Irigaray, T., Schneider, R., \& Gomes, I. (2011). Efeitos de um treino cognitivo na qualidade de vida e no bem-estar psicológico de idosos. Psicologia: Reflexão e Crítica, 24(4), 810-818. 
Kapur, N. (2009). Compensating for memory deficits with memory aids. In B. Wilson (Org), Memory rehabilitation (pp. 54-71). Nova Iorque: The Guilford Press.

Köhler, S., Thomas, A. J., Barnett, N. A., \& O’Brien, J. T. (2010). The pattern and course of cognitive impairment in late-life depression. Psychological Medicine, 40(4), 591-602.

Laks, J., \& Engelhardt, E. (2010). Peculiarities of geriatric psychiatry: a focus on aging and depression. CNS Neuroscience \& Therapeutics, 16(6), 374-379.

Malloy-Diniz, L. F., Cruz, M. F., Torres, V. M., \& Cosenza, R. M. (2000). O teste de Aprendizagem Auditivo-Verbal de Rey: Normas para uma população brasileira. Revista Brasileira de Neurologia, 36(3), 79-83.

Malloy-Diniz, L. F., Lasmar, V. A. P., Gazinelli, L. S. R., Fuentes, D., \& Salgado, J. V. (2007). The Rey Auditory-Verbal Learning Test: applicability for the Brazilian elderly population. Revista Brasileira de Psiquiatria, 29, 324-329.

Minett, T. S. C., Silva, R. V., Ortiz, K. Z., \& Bertolucci, P. H. F. (2008). Subjective memory complaints in an elderly sample: a cross-sectional study. International Journal of Geriatric Psychiatry, 23, 49-54.

Montgomery, S. A., \& Alsberg, M. (1979). A new depression scale designed to be sensitive to change. British Journal of Psychiatry, 134, 382-389.

Nascimento E. (2004). Adaptação, validação e normatização do WAIS-III para uma amostra brasileira. In D. Wechsler (Org.), WAIS-III: Manual para administração e avaliação. São Paulo: Casa do Psicólogo.

Novoa, A. M., Juárez, O., \& Nebot, M. (2008). Review of the effectiveness of cognitive interventions in preventing cognitive deterioration in healthy elderly individuals. Gaceta Sanitaria, 22(5), 474-82.

Rapp, M. A., Dahlman, K., Sano, M., Grossman, H. T., Haroutunian, V., \& Gorman, J. M. (2005). Neuropsychological differences between late-onset and recurrent geriatric major depression. American Journal of Psychiatry, 162, 691-698.

Royall, D. R., Palmer, R., Chiodo, L. K., \& Polk, M. J. (2011). Depressive symptoms predict longitudinal change in executive control but not memory. International Journal of Geriatric Psychiatry, 27(1), 89-96. doi: 10.1002/ gps.2697.

Schoenberg, M. R., Kyra, A. D., Kevin, D., Doyle, P., James, G. S., \& Russell, L. A. (2006.) Test performance and classification statistics for the Rey Auditory Verbal Learning Test in selected clinical samples. Archives of Clinical Neuropsychology, 21, 693-703.

Strauss, E., Sherman, E. M., \& Spreen, O. (2006). A compendium of neuropsychological tests. Nova Iorque: Oxford University.

Tsai, A. Y., Yang, M., Lan, C., \& Chen, C. (2008). Evaluation of effect of cognitive intervention programs for the community-dwelling elderly with subjective memory complaints. International Journal of Geriatric Psychiatry, 23, 1172-1174.

Tulving, E. (1972). Episodic and semantic memory. In E. Tulving, \& W. Donaldson (Orgs.), Organization of memory (pp. 381-403) Nova Iorque: Academic Press.

Unverzagt, F. W., Kasten, L., Johnson, K. E., Rebok, G. W., Marsiske, M., Koepke, K. M., ... \& Tennstedt, S. L. (2007). Effect of memory impairment on training outcomes in ACTIVE. Journal of International Neuropsychological
Society, 13(6), 953-960

World Health Organization [WHO] (2007). Facts on ageing and the life course. Recuperado de <http://www.who.int/features/factfiles/ageing/en/index. html>.

Willis, S. L., Tennstedt, S. L., Marsiske, M., Ball, K., Elias, J., Koepe, K. M., ... ACTIVE Study Group (2006). Long-term effects of cognitive training on everyday functional outcomes in older adults. JAMA, 296(23), 2805-14.

Wilson, B. A. (2009), New learning in rehabilitation: erroless learning, space retrival (expanded reharsal), and vanishing cues. In B. Wilson (Org.), Memory rehabilitation: Integrating theory and practice (pp. 89-105). Nova Iorque: The Guilford Press.

Wilson, B.A., Cockburn, J., Baddeley, A. D., \& Hiorns, R. (1989). The development and validation of a test battery for detecting and monitoring everyday memory problems. Journal of Clinical and Experimental Neuropsychology, 11, 855-870.

Wilson, B. A., \& Evans, J. J. (1996). Error free learning in the rehabilitation of individuals with memory impairments. Journal of Head Trauma Rehabilitation, 11, 54-64.

Wilson, B., \& Gracey, F. (2009). Towards a comprehensive model of neuropsychological rehabilitation. In B. Wilson, F. Gracey, J. Evans, \& A. Bateman (Orgs.), Neuropsychological rehabilitation: Theory, models, therapy and outcome (pp. 1-21). Nova Iorque: Cambridge University Press.

Winocur, G., Palmer, H., Dawson, D., Binns, M. A., Bridges, K., \& Stuss, D. T. (2007). Cognitive rehabilitation in the elderly: an evaluation of psychosocial factors. Journal of the International Neuropsychological Society, 13, 153165.

Wolinsky, F. D., Mahncke, H. W., Weg, M. W. V., Martin, R., Unverzagt, F. W., Ball, K. K., ... \& Tennstedt, S. L. (2009a). The ACTIVE cognitive training interventions and the onset of and recovery from suspected clinical depression. Journal of Gerontology: Psychological Sciences, 64(5), 577-585.

Wolinsky, F. D., Weg, M. W. V., Martin, R., Unverzagt, F. W., Ball, K. K., Jones, R. N., \& Tennstedt, S. L. (2009b). The effect of speed-of-processing training on depressive symptoms in ACTIVE. The Journal of Gerontololy. Series A, Biological Sciences and Medical Sciences, 64(4), 468-472.

Woolley, J. D., Khan, B. K., Murthy, N. K., Miller, B. L., \& Rankin, K. P. (2011). The diagnostic challenge of psychiatric symptoms in neurodegenerative disease: rates of and risk factors for prior psychiatric diagnosis in patients with early neurodegenerative disease. Journal of Clinical Psychiatry, 72(2), 126-133.

Yalom, I., \& Leszcz, M. (2005). Theory and practice of group psychotherapy ( $5^{\text {a }}$ Ed.) (pp. 1-18). Nova Iorque: Basic Books.

Yesavage, J. A., Brink, T. L., Rose, T. L., Lum, O., Huang, V., Adey, M., \& Leirer, V. O. (1983). Development and validation of a geriatric depression screening scale: a preliminary report. Journal of Psychiatric Research, 17, 37-49.

Yassuda, M., Batistoni, S. S., Fortes, A., \& Neri, A. (2006). Treino de memória no idoso saudável: benefícios e mecanismos. Psicologia: Reflexão e Crítica, 19(3), 470-481. 
Tania Maria Netto, doutora em Psicologia Clínica e Neurociências pela Pontifícia Universidade Católica do Rio de Janeiro, é pós-doutoranda em Neurorradiologia pela Universidade Federal do Rio de Janeiro, Departamento de Medicina. Endereço pra correspondência: Rua: Mário Covas Jr. 135/907, Barra da Tijuca, Rio de Janeiro, RJ Telefone: (21) 2493-4039. E-mail: tnetto@yahoo.com

Rochele Paz Fonseca, pós-doutora em Psicologia Clínica e Neurociências pela Pontifícia Universidade Católica do Rio de Janeiro, em Neurorradiologia pela Universidade Federal do Rio de Janeiro e em Ciências Biomédicas pela UdeM. E-mail: rochele.fonseca@gmail.com

Jesus Landeira-Fernandez, doutor em Neurociências e Comportamento pela Universidade da Califórnia em Los Angeles. E-mail:landeira@puc-rio.br 